Proceedings of the Edinburgh Mathematical Society (2002) 45, 205-220 (C)

DOI:10.1017/S0013091500001140 Printed in the United Kingdom

\title{
STABILITY AND BIFURCATION IN A DELAYED RATIO-DEPENDENT PREDATOR-PREY SYSTEM
}

\author{
DONGMEI XIAO* AND WENXIA LI $\dagger$ \\ Department of Mathematics, Central China Normal University, \\ Wuhan 430079, People's Republic of China
}

(Received 6 November 2000)

\begin{abstract}
Recently, ratio-dependent predator-prey systems have been regarded by some researchers as being more appropriate for predator-prey interactions where predation involves serious searching processes. Due to the fact that every population goes through some distinct life stages in real-life, one often introduces time delays in the variables being modelled. The presence of time delay often greatly complicates the analytical study of such models. In this paper, the qualitative behaviour of a class of ratio-dependent predator-prey systems with delay at the equilibrium in the interior of the first quadrant is studied. It is shown that the interior equilibrium cannot be absolutely stable and there exist non-trivial periodic solutions for the model. Moreover, by choosing delay $\tau$ as the bifurcation parameter we study the Hopf bifurcation and the stability of the periodic solutions.
\end{abstract}

Keywords: stability; bifurcation; predator-prey system; ratio-dependent response; time delay

AMS 2000 Mathematics subject classification: Primary 34C25; 92D25

Secondary $58 \mathrm{~F} 14$

\section{Introduction}

In population dynamics, the models most often used by ecologists to describe interactions between predator and prey populations are variations of the standard Lotka-Volterratype models. The standard Lotka-Volterra model is built by assuming that the per capita rate of predation depends on the prey numbers only. Recently, the traditional prey-dependent predator-prey models have been challenged by several biologists (see $[\mathbf{1}-\mathbf{3}, \mathbf{1 0}]$ and the references cited therein) based on the fact that functional and numerical responses over typical ecological time-scales ought to depend on the densities of both prey and predators, especially when predators have to share or compete for food. Such a functional response is called ratio-dependent; roughly stated, the per capita predator growth rate should be a function of the ratio of prey to predator abundance. Thus an alternative assumption is that, as the numbers of predators change slowly (relative to

* Present address: Department of Mathematics, Shanghai Jiaotong University, Shanghai 200030, People's Republic of China (xiaodm@sjtu.edu.cn).

$\dagger$ Present address: Department of Mathematics, East China Normal University, Shanghai 200062, People's Republic of China (wxli@math.ecnu.edu.cn). 
prey change), the per capita rate of predation depends on the numbers of both prey and predator, most probably and simply on their ratio, since there is often competition among the predators. Based on the Michaelis-Menten or Holling type II function, Arditi and Ginzburg [2] proposed a ratio-dependent function of the form

$$
p\left(\frac{x}{y}\right)=\frac{c(x / y)}{m+(x / y)}=\frac{c x}{m y+x}
$$

and the following ratio-dependent predator-prey model:

$$
\left.\begin{array}{c}
\dot{x}=x(a-b x)-\frac{c x y}{m y+x}, \\
\dot{y}=y\left(-d+\frac{f x}{m y+x}\right) .
\end{array}\right\}
$$

Here $x(t)$ and $y(t)$ represent the population densities of prey and predator at time $t$, respectively; $(a / b)>0$ is the carrying capacity of the prey, $d>0$ is the death rate of the predator, and $a, c, m$ and $f$ are positive constants that stand for prey intrinsic growth rate, capturing rate, half saturation constant and conversion rate, respectively.

As is typical for predator-prey systems, the $x$-axis, $y$-axis and the interior of the first quadrant are all invariant under system (1.1), and solutions with positive initial values are positive and bounded. However, differing from the prey-dependent predator-prey models, the ratio-dependent predator-prey systems have two principal predictions:

(a) equilibrium abundances are positively correlated along a gradient of enrichment (see Arditi and Gurzburg [2]); and

(b) the 'paradox of enrichment' (see Rosenzweig [16]) either completely disappears or enrichment is linked to stability in a more complex way.

The ratio-dependent predator-prey model (1.1) has been studied by many researchers recently and very rich dynamics have been observed (see, for example, $[\mathbf{1 0}-\mathbf{1 3}, \mathbf{1 5}, \mathbf{1 7}]$ ). On the other hand, both ecologists and mathematicians often introduce time delays in the variables being modelled due to the fact that every population goes through some distinct life stages in real-life problems. In [4] Beretta and Kuang proposed a ratiodependent model with a single discrete positive delay $\tau$ :

$$
\left.\begin{array}{l}
\dot{x}(t)=x(t)(a-b x(t))-\frac{c x(t) y(t)}{m y(t)+x(t)}, \\
\dot{y}(t)=y(t)\left(-d+\frac{f x(t-\tau)}{m y(t-\tau)+x(t-\tau)}\right) .
\end{array}\right\}
$$

The system (1.2) satisfies the theorem of existence and uniqueness of solutions under the initial-value condition

$$
x_{0}(\theta)=\phi_{1}(\theta) \geqslant 0, \quad y_{0}(\theta)=\phi_{2}(\theta) \geqslant 0, \quad \theta \in[-\tau, 0], \quad x_{0}(0)>0, \quad y_{0}(0)>0,
$$


where $\phi=\left(\phi_{1}, \phi_{2}\right) \in C\left([-\tau, 0], R_{+}^{2}\right), R_{+}^{2}=\{(x, y): x \geqslant 0, y \geqslant 0\}$ and $\|\phi\|=$ $\max \{|\phi(\theta)|: \theta \in[-\tau, 0]\}$ with $|\phi|$ any norm in $R^{2}$.

Beretta and Kuang in [4] made use of a rather novel and non-trivial way of constructing proper Lyapunov functions to obtain some new and significant global stability or convergence results for (1.2). However, some subtle mathematical questions on the behaviour of solutions of the model are far from completely answered: for example, the existence of periodic solutions for the model.

In this paper, we pay attention to the interior equilibrium of (1.2). First we study the effect of time delay on local stability of the interior equilibrium, then we investigate conditions on the delay and parameters of (1.2) so that the interior equilibrium is conditionally stable or unstable. It will also be shown that the interior equilibrium of (1.2) cannot be absolutely stable for all parameters.

We then apply the method in [9] to analyse Hopf bifurcation of (1.2) by choosing delay $\tau$ as a bifurcation parameter, and show the existence and stability of periodic solutions to $(1.2)$.

This paper is organized as follows. In the next section we show that delay does cause instability, and we present results on non-occurrence of absolute stability and on conditional stability of the interior equilibrium. In $\S 3$, the analysis of singularities is performed by using the normal form theory for functional differential equations in [9] and choosing delay $\tau$ as a bifurcation parameter. We show that when the delay takes some critical values the interior equilibrium becomes unstable and Hopf bifurcation occurs, i.e. periodic solutions bifurcate from the interior equilibrium as delay $\tau$ passes through the critical values. We give the conditions which guarantee the stability of the periodic solution and use an example to illustrate the result. The paper ends with a brief discussion.

\section{Local stability analysis}

In this section, we focus on investigating the local stability of the interior equilibrium of the system (1.2). As shown in [14], (1.2) (or (1.1)) has a unique interior equilibrium if and only if any one of the following two conditions is true:

(1) $d<f$ and $c \leqslant m a$; or

(2) $d<f<(c d /(c-a m))$ and $a m<c$.

We denote this unique interior equilibrium by $E^{*}=\left(x^{*}, y^{*}\right)$ and use this notation throughout this paper, here

$$
x^{*}=\frac{a}{b}-\frac{c(f-d)}{b m f}, \quad y^{*}=\frac{f-d}{d m} x^{*} .
$$

To study the local stability of the interior equilibrium $E^{*}$, we consider linearization of $(1.2)$ at $E^{*}\left(x^{*}, y^{*}\right)$ :

$$
\left.\begin{array}{l}
\dot{X}(t)=\alpha_{1} X(t)+\alpha_{2} Y(t) \\
\dot{Y}(t)=\beta_{1} X(t-\tau)+\beta_{2} Y(t-\tau),
\end{array}\right\}
$$


where

$$
\begin{array}{ll}
\alpha_{1}=-b x^{*}+\frac{c x^{*} y^{*}}{\left(m y^{*}+x^{*}\right)^{2}}, & \alpha_{2}=-\frac{c x^{* 2}}{\left(m y^{*}+x^{*}\right)^{2}}, \\
\beta_{1}=\frac{f m y^{* 2}}{\left(m y^{*}+x^{*}\right)^{2}}, & \beta_{2}=-\frac{f m x^{*} y^{*}}{\left(m y^{*}+x^{*}\right)^{2}} .
\end{array}
$$

The characteristic equation for (2.1) takes the form

$$
\lambda^{2}-\alpha_{1} \lambda-\beta_{2} \lambda \mathrm{e}^{-\lambda \tau}+\left(\alpha_{1} \beta_{2}-\alpha_{2} \beta_{1}\right) \mathrm{e}^{-\lambda \tau}=0,
$$

where $\alpha_{1} \beta_{2}-\alpha_{2} \beta_{1}>0$.

We recall some definitions (see $[\mathbf{5}-\mathbf{7}, \mathbf{1 2}])$. The equilibrium $\left(x^{*}, y^{*}\right)$ is called asymptotically stable if there exists a $\delta>0$ such that

$$
\sup _{-\tau \leqslant \theta \leqslant 0}\left[\left|\phi_{1}(\theta)-x^{*}\right|+\left|\phi_{2}(\theta)-y^{*}\right|\right]<\delta
$$

implies that

$$
\lim _{t \rightarrow \infty}(x(t), y(t))=\left(x^{*}, y^{*}\right),
$$

where $(x(t), y(t))$ is the solution of (1.2) which satisfies the condition (1.3). The equilibrium $\left(x^{*}, y^{*}\right)$ is absolutely stable if it is asymptotically stable for all delays $\tau \geqslant 0$ and is conditionally stable if it is asymptotically stable for $\tau$ in some finite interval. The equilibrium $\left(x^{*}, y^{*}\right)$ is absolutely unstable if it is unstable for all delay $\tau \geqslant 0$.

It is known that the equilibrium is asymptotically stable if all roots of the corresponding characteristic equation have negative real parts. For the equilibrium $E^{*}$ of (1.2) there are two types of stability: absolute stability (independent of the delay) and conditional stability (depending on the delay). We know that the time delay cannot change the number and location of equilibria of (1.2). Hence (1.2) has the same equilibria as the corresponding ordinary differential equation (ODE) system (i.e. the system (1.1)). The equilibrium $E^{*}$ of (1.2) is absolutely stable if and only if the equilibrium $E^{*}$ of the corresponding ODE system (i.e. system (1.1)) is asymptotically stable and the characteristic equation (2.2) has no purely imaginary roots for any $\tau>0$. Thus if all roots of the characteristic equation (2.2) have negative real parts at $\tau=0$ and there exist some positive values $\tau$ such that the characteristic equation (2.2) has a pair of purely imaginary roots, say $\pm \mathrm{i} \omega_{0}$, then the equilibrium $E^{*}$ of $(1.2)$ is not absolutely stable but could be conditionally stable. Suppose that $\omega_{0}$ is obtained when the delay reaches a positive value $\tau_{0}$, where $\tau_{0}$ satisfies the condition that when $0<\tau<\tau_{0}$ the real parts of all roots of the characteristic equation still remain negative, and when $\tau=\tau_{0}$ the characteristic equation (2.2) has a pair of purely imaginary roots $\pm \mathrm{i} \omega_{0}$. If, moreover, the transversal condition holds at $\tau=\tau_{0}$, then when $\tau>\tau_{0}$ the characteristic equation (2.2) will have at least one root with positive real part and the equilibrium $E^{*}$ of (1.2) becomes unstable by Rouché's theorem [8]. Hence, the equilibrium $E^{*}$ of (1.2) is conditionally stable.

In order to study the local stability of (1.2), we have to recall some results of the corresponding ODE system (i.e. (1.1)) on stability of the interior equilibrium $E^{*}$. The proof of the following lemma can be found in $[\mathbf{1 7}]$. 
Lemma 2.1. Suppose that (1.1) has a unique interior equilibrium $\left(x^{*}, y^{*}\right)$, i.e. either one of conditions (1) and (2) holds. Denote $\Delta=m^{2} d^{4}+4 c d^{2}(c-a m-d m)$. Then there are only three possibilities.

(1) The equilibrium $\left(x^{*}, y^{*}\right)$ of (1.1) is locally asymptotically stable if any of the following conditions hold:

(i) $d<f$ and $c \leqslant m a$;

(ii) $d<f<(c d /(c-a m))$ and $a m<c \leqslant a m+d m$;

(iii) $d<f<\left[\left(-m d^{2}+\sqrt{\Delta}\right) /(2(c-a m-d m))\right]$ and $0<c-a m-d m$.

(2) The equilibrium $\left(x^{*}, y^{*}\right)$ is unstable if

(iv) $\left[\left(-m d^{2}+\sqrt{\Delta}\right) /(2(c-a m-d m))\right]<f<(c d /(c-a m))$ and $0<c-a m-d m$.

(3) The equilibrium $\left(x^{*}, y^{*}\right)$ is non-hyperbolic if

(v) $f=\left[\left(-m d^{2}+\sqrt{\Delta}\right) /(2(c-a m-d m))\right]$ and $0<c-a m-d m$.

Remark 2.2. According to the results in [14], the limit cycle of (1.1) is unique and stable if it exists. We find that the non-hyperbolic equilibrium $\left(x^{*}, y^{*}\right)$ in case (3) is stable if a non-trivial periodic orbit can be bifurcated by $\left(x^{*}, y^{*}\right)$.

By applying the theorem from Kuang [12], we see that if $\left(x^{*}, y^{*}\right)$ is unstable for (1.1), then it will remain so for all $\tau>0$. Hence, from Lemma 2.1 we have the following theorem.

Theorem 2.3. When $\left[\left(-m d^{2}+\sqrt{\Delta}\right) /(2(c-a m-d m))\right]<f<(c d /(c-a m))$ and $0<c-a m-d m$ with $\Delta=m^{2} d^{4}+4 c d^{2}(c-a m-d m)$, the interior equilibrium $\left(x^{*}, y^{*}\right)$ of (1.2) is absolutely unstable.

We now turn to an investigation of the roots of the characteristic equation (2.2). Obviously, these roots depend on $\tau$. In the following we explore the case when (2.2) has a pair of purely imaginary roots. Assume that for some $\tau \geqslant 0$, i $\omega$ with $\omega>0$ is a root of (2.2). We then have

$$
-\omega^{2}-\alpha_{1} \omega \mathrm{i}-\beta_{2} \omega \mathrm{ie}^{-\omega \tau \mathrm{i}}+\left(\alpha_{1} \beta_{2}-\alpha_{2} \beta_{1}\right) \mathrm{e}^{-\omega \tau \mathrm{i}}=0 .
$$

Separating the real and imaginary parts, we obtain

$$
\left.\begin{array}{r}
-\omega^{2}-\beta_{2} \omega \sin \omega \tau+\left(\alpha_{1} \beta_{2}-\alpha_{2} \beta_{1}\right) \cos \omega \tau=0, \\
-\alpha_{1} \omega-\beta_{2} \omega \cos \omega \tau-\left(\alpha_{1} \beta_{2}-\alpha_{2} \beta_{1}\right) \sin \omega \tau=0 .
\end{array}\right\}
$$

Equations (2.3) are equivalent to

$$
\left.\begin{array}{l}
\cos \omega \tau=-\frac{\alpha_{2} \beta_{1} \omega^{2}}{\beta_{2}^{2} \omega^{2}+\left(\alpha_{1} \beta_{2}-\alpha_{2} \beta_{1}\right)^{2}}, \\
\sin \omega \tau=-\frac{\beta_{2} \omega^{3}+\alpha_{1} \omega\left(\alpha_{1} \beta_{2}-\alpha_{2} \beta_{1}\right)}{\beta_{2}^{2} \omega^{2}+\left(\alpha_{1} \beta_{2}-\alpha_{2} \beta_{1}\right)^{2}} .
\end{array}\right\}
$$


From Equations (2.4) we have

$$
\left(-\frac{\alpha_{2} \beta_{1} \omega^{2}}{\beta_{2}^{2} \omega^{2}+\left(\alpha_{1} \beta_{2}-\alpha_{2} \beta_{1}\right)^{2}}\right)^{2}+\left(-\frac{\beta_{2} \omega^{3}+\alpha_{1} \omega\left(\alpha_{1} \beta_{2}-\alpha_{2} \beta_{1}\right)}{\beta_{2}^{2} \omega^{2}+\left(\alpha_{1} \beta_{2}-\alpha_{2} \beta_{1}\right)^{2}}\right)^{2}=1 .
$$

Thus if (2.5) has positive real solutions, then we can get the corresponding $\tau \geqslant 0$ from (2.4) such that the characteristic equation (2.2) has purely imaginary roots. It is not difficult to check that (2.5) does have positive real roots by letting $s=\omega^{2}$ to change (2.5) into a polynomial equation in $s$. Furthermore, in order to determine the exact value of $\omega$, by (2.3) we have

$$
\omega^{4}+\left(\alpha_{1}^{2}-\beta_{2}^{2}\right) \omega^{2}-\left(\alpha_{1} \beta_{2}-\alpha_{2} \beta_{1}\right)^{2}=0
$$

which has a unique positive real root

$$
\omega_{0}=\sqrt{\frac{1}{2}\left(-\left(\alpha_{1}^{2}-\beta_{2}^{2}\right)+\sqrt{\left(\alpha_{1}^{2}-\beta_{2}^{2}\right)^{2}+4\left(\alpha_{1} \beta_{2}-\alpha_{2} \beta_{1}\right)^{2}}\right)}
$$

Therefore, we have the following lemma.

Lemma 2.4. Let $\Delta=m^{2} d^{4}+4 c d^{2}(c-a m-d m)$.

(1) Suppose that any one of the following conditions hold:

(i) $d<f$ and $c \leqslant m a$;

(ii) $d<f<(c d /(c-a m))$ and $a m<c \leqslant a m+d m$;

(iii) $d<f<\left[\left(-m d^{2}+\sqrt{\Delta}\right) /(2(c-a m-d m))\right]$ and $0<c-a m-d m$.

Then, only when

$$
\tau=\tau_{k}=\omega_{0}^{-1} \arcsin \left(-\frac{\beta_{2} \omega_{0}^{3}+\alpha_{1} \omega_{0}\left(\alpha_{1} \beta_{2}-\alpha_{2} \beta_{1}\right)}{\beta_{2}^{2} \omega_{0}^{2}+\left(\alpha_{1} \beta_{2}-\alpha_{2} \beta_{1}\right)^{2}}\right)+2 k \pi
$$

for some $k=0,1,2, \ldots$ (or some $k=1,2, \ldots)$ if $\tau_{0}>0$ (respectively, $\tau_{0}<0$ ), does the characteristic equation (2.2) have a unique pair of purely imaginary roots $\pm \mathrm{i} \omega_{0}$, where $\omega_{0}$ is given by (2.6).

(2) If $f=\left[\left(-m d^{2}+\sqrt{\Delta}\right) /(2(c-a m-d m))\right]$ and $0<c-a m-d m$, then only when $\tau=\tau_{k}^{*}=2 k \pi / \omega_{0}$ for some $k=0,1,2, \ldots$, does the characteristic equation (2.2) have a unique pair of purely imaginary roots $\pm \mathrm{i} \omega_{0}$, where $\omega_{0}=\sqrt{\alpha_{1} \beta_{2}-\alpha_{2} \beta_{1}}$.

Proof. (1) From the above analysis, we can see that the characteristic equation (2.2) only has a unique pair of purely imaginary roots $\pm \mathrm{i} \omega_{0}$ when the non-negative real number $\tau$ satisfies Equations (2.4). Noting that $-\alpha_{2} \beta_{1}>0$, from (2.4) we get

$$
\tau=\tau_{k}=\omega_{0}^{-1} \arcsin \left(-\frac{\beta_{2} \omega_{0}^{3}+\alpha_{1} \omega_{0}\left(\alpha_{1} \beta_{2}-\alpha_{2} \beta_{1}\right)}{\beta_{2}^{2} \omega_{0}^{2}+\left(\alpha_{1} \beta_{2}-\alpha_{2} \beta_{1}\right)^{2}}\right)+2 k \pi .
$$


Here we would like to point out that $\tau_{0} \neq 0$ when one of the conditions (i)-(iii) holds. Hence, to guarantee $\tau_{k}>0$ we take $k=0,1,2 \ldots($ or $k=1,2, \ldots)$ if $\tau_{0}>0$ (respectively, $\left.\tau_{0}<0\right)$.

(2) Note that $f=\left[\left(-m d^{2}+\sqrt{\Delta}\right) /(2(c-a m-d m))\right]$ implies $\alpha_{1}+\beta_{2}=0$. So we have $\omega_{0}=\sqrt{\alpha_{1} \beta_{2}-\alpha_{2} \beta_{1}}$ by (2.6). Correspondingly, from (2.4) we get $\tau=\tau_{k}^{*}=2 k \pi / \omega_{0}$, $k=0,1,2, \ldots$.

Now we investigate how the real part of the roots of (2.2) varies as $\tau$ varies in a small neighbourhood of $\tau_{k}$. Let $u+\mathrm{i} \omega$ be a root of (2.2). Separating the real and imaginary parts of (2.2) we then have

$$
\left.\begin{array}{l}
H_{1}(u, \omega, \tau)=0, \\
H_{2}(u, \omega, \tau)=0,
\end{array}\right\}
$$

where

$H_{1}(u, \omega, \tau)=u^{2}-\omega^{2}-\alpha_{1} u-\mathrm{e}^{-u \tau} \beta_{2} u \cos \omega \tau-\mathrm{e}^{-u \tau} \beta_{2} \omega \sin \omega \tau+\left(\alpha_{1} \beta_{2}-\alpha_{2} \beta_{1}\right) \mathrm{e}^{-u \tau} \cos \omega \tau$

and

$H_{2}(u, \omega, \tau)=2 u \omega-\alpha_{1} \omega-\mathrm{e}^{-u \tau} \beta_{2} \omega \cos \omega \tau+\mathrm{e}^{-u \tau} \beta_{2} u \sin \omega \tau-\left(\alpha_{1} \beta_{2}-\alpha_{2} \beta_{1}\right) \mathrm{e}^{-u \tau} \sin \omega \tau$.

By Lemma 2.4 we have $H_{1}\left(0, \omega_{0}, \tau_{k}\right)=H_{2}\left(0, \omega_{0}, \tau_{k}\right)=0$. It is easy to check that the Jacobian matrix

$$
J=\left(\begin{array}{ll}
\frac{\partial H_{1}}{\partial u} & \frac{\partial H_{1}}{\partial \omega} \\
\frac{\partial H_{2}}{\partial u} & \frac{\partial H_{2}}{\partial \omega}
\end{array}\right)
$$

satisfies $|J|_{\left(0, \omega_{0}, \tau_{k}\right)}>0$. By means of the implicit function theorem we deduce that Equations (2.7) define $u, \omega$ as functions of $\tau$ in a neighbourhood of $\left(0, \omega_{0}, \tau_{k}\right)$ such that $u\left(\tau_{k}\right)=0$ and $\omega\left(\tau_{k}\right)=\omega_{0}$. Moreover, $\mathrm{d} u\left(\tau_{k}\right) / \mathrm{d} t>0$. Thus this root of $(2.2)$ crosses the imaginary axis from the left to the right as $\tau$ continuously varies from a number less than $\tau_{k}$ to one greater than $\tau_{k}$ by Rouché's theorem and Lemma 2.1. The following theorem is an addition to Theorem 2.3, which can be proven by the above arguments and Lemma 2.4.

Theorem 2.5. Let $\Delta=m^{2} d^{4}+4 c d^{2}(c-a m-d m)$.

(1) When any one of the following conditions holds,

(i) $d<f$ and $c \leqslant m a$,

(ii) $d<f<(c d /(c-a m))$ and $a m<c \leqslant a m+d m$,

(iii) $d<f<\left[\left(-m d^{2}+\sqrt{\Delta}\right) /(2(c-a m-d m))\right]$ and $0<c-a m-d m$,

the interior equilibrium $\left(x^{*}, y^{*}\right)$ of $(1.2)$ is conditionally stable. Thus a small variation of delay does not change the stability of the interior equilibrium. 
(2) When $f=\left[\left(-m d^{2}+\sqrt{\Delta}\right) /(2(c-a m-d m))\right]$ and $0<c-a m-d m$, the interior equilibrium $\left(x^{*}, y^{*}\right)$ of (1.2) is unstable for all $\tau>0$.

Remark 2.6. From Remark 2.2 we can see that any small delay can change the stability of the interior equilibrium in case (2) of Theorem 2.5 .

Remark 2.7. Theorems 2.3 and 2.5 imply the non-occurrence of absolute stability of the interior equilibrium $\left(x^{*}, y^{*}\right)$ of $(1.2)$.

\section{The Hopf bifurcation}

In this section we will discuss the Hopf bifurcation of (1.2) where we take $\tau$ as a bifurcation parameter. According to the analysis in $\S 2$, one can see that when the interior equilibrium $\left(x^{*}, y^{*}\right)$ of (1.1) is locally asymptotically stable, the characteristic equation (2.2) of (1.2) has pure imaginary roots $\pm \mathrm{i} \omega_{0}$ if and only if $\tau=\tau_{k}$, and the transversal condition holds at $\tau=\tau_{k}$. Thus the following theorem is implied by Theorem 2.5 (see [12]).

Theorem 3.1. If any one of the following conditions holds,

(i) $d<f$ and $c \leqslant m a$,

(ii) $d<f<(c d /(c-a m))$ and $a m<c \leqslant a m+d m$,

(iii) $d<f<\left[\left(-m d^{2}+\sqrt{\Delta}\right) /(2(c-a m-d m))\right]$ and $0<c-a m-d m$, where $\Delta=$ $m^{2} d^{4}+4 c d^{2}(c-a m-d m)$,

then a Hopf bifurcation occurs on a two-dimensional centre manifold for (1.2) at the interior equilibrium $\left(x^{*}, y^{*}\right)$ for $\tau=\tau_{k}$ (here $k \in N_{0}=\{0,1,2, \ldots\}$ or $k \in N=\{1,2, \ldots\}$ as stated in Lemma 2.4), which is associated with the simple eigenvalues $\pm \mathrm{i} \omega_{0}$. This centre manifold is locally stable for $\tau=\tau_{0}$ (or, respectively, $\tau=\tau_{1}$ ) and unstable for $\tau=\tau_{k}$, $k=1,2, \ldots$ (or, respectively, $k=2,3, \ldots$ ).

To determine the direction of the bifurcation and the stability of the periodic solution created by Hopf bifurcation, the normal form theory for functional differential equations (FDEs) developed by Faria and Magalhães [9] will be employed for discussing the explicit expressions of the normal form of (1.2) in terms of the original parameters in a small neighbourhood of $\tau_{k}$. By time scaling $t \rightarrow(t / \tau),(1.2)$ is transformed into

$$
\left.\begin{array}{l}
\dot{x}(t)=\tau\left(x(t)(a-b x(t))-\frac{c x(t) y(t)}{m y(t)+x(t)}\right) \triangleq \tau f^{(1)}(x(t), y(t)), \\
\dot{y}(t)=\tau y(t)\left(-d+\frac{f x(t-1)}{m y(t-1)+x(t-1)}\right) \triangleq \tau f^{(2)}(y(t), x(t-1), y(t-1)),
\end{array}\right\}
$$

in a fixed phase space $C_{1}=C\left([-1,0] ; R_{+}^{2}\right)$.

Even though the normal form procedure for FDEs is given in $[\mathbf{9}]$, it is very difficult to completely treat (3.1) for all permissible parameters since hard computation is involved.

To simplify computation, we only consider the Hopf bifurcation of (3.1) in the case that $f=\sqrt{c d^{2} /(c-a m)}$. In this case, we have $\alpha_{1}=0$ in (2.1). 
Theorem 3.2. Suppose $a m<c<a m+d m$ and $f=\sqrt{c d^{2} /(c-a m)}$. Then (3.1) has a unique interior equilibrium $\left(x_{0}, y_{0}\right)$, where

$$
x_{0}=\frac{a}{b}-\frac{c-\sqrt{c^{2}-a m c}}{b m}, \quad y_{0}=\frac{\sqrt{c}-\sqrt{c-a m}}{m \sqrt{c-a m}} x_{0} .
$$

Furthermore, there exists a $\tau^{(0)}$ such that $\left(x_{0}, y_{0}\right)$ is locally asymptotically stable (unstable) whenever $0<\tau<\tau^{(0)}$ (respectively, $\tau>\tau^{(0)}$ ). When $\tau=\tau^{(k)}$, there exists a unique pair of simple purely imaginary roots $\pm \mathrm{i} \omega_{k}$ for the characteristic equation of the linearized equation of (3.1) around $\left(x_{0}, y_{0}\right)$, where

$$
\begin{aligned}
\tau^{(k)} & =\sqrt{\frac{\omega_{k}^{2}\left(\sqrt{b_{2}^{4}+4 a_{2}^{2} b_{1}^{2}}-b_{2}^{2}\right)}{2 a_{2}^{2} b_{1}^{2}}}, \quad \omega_{k}=\arccos \frac{b_{2}^{2}-\sqrt{b_{2}^{4}+4 a_{2}^{2} b_{1}^{2}}}{2 a_{2} b_{1}}+2 k \pi, \quad k=0,1,2, \ldots, \\
a_{2} & =-\frac{c\left(x_{0}\right)^{2}}{\left(m y_{0}+x_{0}\right)^{2}}, \quad b_{1}=\frac{m\left(y_{0}\right)^{2}}{\left(m y_{0}+x_{0}\right)^{2}} \sqrt{\frac{c d^{2}}{c-a m}}, \quad b_{2}=-\frac{m x_{0} y_{0}}{\left(m y_{0}+x_{0}\right)^{2}} \sqrt{\frac{c d^{2}}{c-a m}} .
\end{aligned}
$$

Proof. First of all, letting $x_{1}=x-x_{0}, x_{2}=y-y_{0}$, we move the equilibrium $\left(x_{0}, y_{0}\right)$ of $(3.1)$ to the origin. By noting that $f=\sqrt{c d^{2} /(c-a m)}$ and separating the linear terms from the nonlinear terms, (3.1) can be changed into

$$
\left.\begin{array}{l}
\dot{x}_{1}(t)=\tau\left(a_{2} x_{2}(t)+F_{1}\left(x_{1}(t), x_{2}(t)\right)\right), \\
\dot{x}_{2}(t)=\tau\left(b_{1} x_{1}(t-1)+b_{2} x_{2}(t-1)+F_{2}\left(x_{2}(t), x_{1}(t-1), x_{2}(t-1)\right)\right),
\end{array}\right\}
$$

where

$$
a_{2}=-\frac{c\left(x_{0}\right)^{2}}{\left(m y_{0}+x_{0}\right)^{2}}, \quad b_{1}=\frac{m\left(y_{0}\right)^{2}}{\left(m y_{0}+x_{0}\right)^{2}} \sqrt{\frac{c d^{2}}{c-a m}}, \quad b_{2}=-\frac{m x_{0} y_{0}}{\left(m y_{0}+x_{0}\right)^{2}} \sqrt{\frac{c d^{2}}{c-a m}}
$$

and

$$
\begin{gathered}
F_{1}\left(x_{1}(t), x_{2}(t)\right)=f^{(1)}\left(x_{1}(t)+x_{0}, x_{2}(t)+y_{0}\right)-\frac{\partial f^{(1)}\left(x_{0}, y_{0}\right)}{\partial x} x_{1}(t)-\frac{\partial f^{(1)}\left(x_{0}, y_{0}\right)}{\partial y} x_{2}(t), \\
F_{2}\left(x_{2}(t), x_{1}(t-1), x_{2}(t-1)\right) \\
=f^{(2)}\left(x_{2}(t)+y_{0}, x_{1}(t-1)+x_{0}, x_{2}(t-1)+y_{0}\right)-\frac{\partial f^{(2)}\left(y_{0}, x_{0}, y_{0}\right)}{\partial y} x_{2}(t) \\
-\frac{\partial f^{(2)}\left(y_{0}, x_{0}, y_{0}\right)}{\partial x(t-1)} x_{1}(t-1)-\frac{\partial f^{(2)}\left(y_{0}, x_{0}, y_{0}\right)}{\partial y(t-1)} x_{2}(t-1) .
\end{gathered}
$$

To study the local stability of the zero solution of (3.2), we consider the characteristic equation of the linearized equation

$$
\left.\begin{array}{l}
\dot{x}_{1}(t)=\tau a_{2} x_{2}(t) \\
\dot{x}_{2}(t)=\tau\left(b_{1} x_{1}(t-1)+b_{2} x_{2}(t-1)\right)
\end{array}\right\}
$$

around the equilibrium $(0,0)$, which is given by

$$
\Delta(\lambda, \tau)=\lambda^{2}-b_{2} \tau \lambda \mathrm{e}^{-\lambda}-a_{2} b_{1} \tau^{2} \mathrm{e}^{-\lambda}=0 .
$$


Assume that for some $\tau>0$, Equation (3.4) has a root $i \omega, \omega>0$. Then

$$
\left.\begin{array}{r}
-\omega^{2}-b_{2} \tau \omega \sin \omega-a_{2} b_{1} \tau^{2} \cos \omega=0, \\
-b_{2} \tau \omega \cos \omega+a_{2} b_{1} \tau^{2} \sin \omega=0 .
\end{array}\right\}
$$

It is easy to see that Equations (3.5) are equivalent to

$$
\left.\begin{array}{r}
-\omega^{2} \cos \omega-a_{2} b_{1} \tau^{2}=0 \\
-b_{2} \tau-\omega \sin \omega=0
\end{array}\right\}
$$

Solving Equations (3.6) yields the following solutions:

$$
\omega_{k}=\arccos \frac{b_{2}^{2}-\sqrt{b_{2}^{4}+4 a_{2}^{2} b_{1}^{2}}}{2 a_{2} b_{1}}+2 k \pi, \quad \tau^{(k)}=\sqrt{\frac{\omega_{k}^{2}\left(\sqrt{b_{2}^{4}+4 a_{2}^{2} b_{1}^{2}}-b_{2}^{2}\right)}{2 a_{2}^{2} b_{1}^{2}}}, \quad k=0,1, \ldots
$$

As a result, (3.4) has the root $\mathrm{i} \omega_{k}$ if and only if $\tau=\tau^{(k)}$.

Now let $H_{1}(u, \omega, \tau)=\operatorname{Re} \Delta(u+\mathrm{i} \omega, \tau), H_{2}(u, \omega, \tau)=\operatorname{Im} \Delta(u+\mathrm{i} \omega, \tau)$ and $H(u, \omega, \tau)=$ $\left(H_{1}(u, \omega, \tau), H_{2}(u, \omega, \tau)\right)$. By the implicit function theorem, in a small neighbourhood of $\tau^{(k)}$ the equation $H(u, \omega, \tau)=0$ defines $u, \omega$ as functions of $\tau$ such that $u\left(\tau^{(k)}\right)=0$, $\omega\left(\tau^{(k)}\right)=\omega_{k}$ and $u^{\prime}\left(\tau^{(k)}\right)>0$. Thus the root $\omega_{k} \mathrm{i}$ of equation $\Delta\left(\lambda, \tau^{(k)}\right)=0$ is simple. Using a similar method, we can prove that the root $-\mathrm{i} \omega_{k}$ of $\Delta\left(\lambda, \tau^{(k)}\right)=0$ is simple too. This completes the proof of the theorem.

We now introduce some notation. Considering the phase space $C_{1},(3.2)$ can be written as an equation in $C_{1}$

$$
\dot{X}(t)=\tau L\left(X_{t}\right)+\tau F\left(X_{t}\right)
$$

where $X(t)$ is a vector $\left(x_{1}(t), x_{2}(t)\right), X_{t} \in C_{1}, X_{t}(\theta)=X(t+\theta),-1 \leqslant \theta \leqslant 0$, and $L: C_{1} \rightarrow R_{+}^{2}, F: C_{1} \rightarrow R_{+}^{2}$ are given by

$$
L(\varphi)=\left(\begin{array}{c}
a_{2} \varphi_{2}(0) \\
b_{1} \varphi_{1}(-1)+b_{2} \varphi_{2}(-1)
\end{array}\right), \quad F(\varphi)=\left(\begin{array}{c}
F_{1}\left(\varphi_{1}(0), \varphi_{2}(0)\right) \\
F_{2}\left(\varphi_{2}(0), \varphi_{1}(-1), \varphi_{2}(-1)\right)
\end{array}\right)
$$

for $\varphi=\left(\varphi_{1}, \varphi_{2}\right) \in C_{1}$.

Let $A$ be the generator of the linear semigroup corresponding to (3.3). When $\tau=\tau^{(k)}, A$ has a pair of simple purely imaginary characteristic roots $\pm \mathrm{i} \omega_{k}$ and no other characteristic roots with zero real part. Hence, Hopf bifurcation may occur at $\tau=\tau^{(k)}$.

For a fixed $k \in N_{0}$, define $\Lambda=\left\{-\mathrm{i} \omega_{k}, \mathrm{i} \omega_{k}\right\}$ and introduce a new parameter $\nu=\tau-\tau^{(k)}$. The system (3.7) can be written as

$$
\dot{X}(t)=\tau^{(k)} L\left(X_{t}\right)+F_{0}\left(X_{t}, \nu\right)
$$

where $F_{0}(\varphi, \nu)=\nu L(\varphi)+\left(\tau^{(k)}+\nu\right) F(\varphi)$.

To describe the Hopf bifurcation occurring at $\tau^{(k)}$ and the stability of the periodic solutions by Hopf bifurcation, we will apply the normal form theory for FDEs in $[\mathbf{9}]$ 
to (3.8). Let the phase space $C_{1}$ be decomposed by $\Lambda$ as $C_{1}=P \oplus Q$, where $P$ is the generalized eigenspace associated with $\Lambda$. Consider the adjoint bilinear form $(\cdot, \cdot)$ on $C_{1}^{*} \times C_{1}$ associated with the linear equation $\dot{X}(t)=\tau_{k} L\left(X_{t}\right)$ :

$$
(\psi, \phi)=\psi(0) \phi(0)-\int_{-r}^{0} \int_{0}^{\theta} \psi(\xi-\theta) \mathrm{d} \eta(\theta) \phi(\xi) \mathrm{d} \xi
$$

Assume that $\Phi=\left(\phi_{1}, \phi_{2}\right)$ is a basis for $P$ and $\Psi=\operatorname{col}\left(\psi_{1}, \psi_{2}\right)$ is a basis for the dual space $P^{*}$ in $C_{1}^{*}$ associated with the eigenvalues $\pm \mathrm{i} \omega_{k}$ of the adjoint equations. Then it can be normalized so that $(\Phi, \Psi)=I$. Here it is convenient to combine one complex coordinate and two complex conjugate basis vectors to describe a two-dimensional real subspace $P$. Consider $(3.8)$ in $C([-1,0] ; \mathbb{C})$, still denoted by $C_{1}$. Note that $\dot{\Phi}=\Phi B$, where $B=\operatorname{diag}\left(\mathrm{i} \omega_{k},-\mathrm{i} \omega_{k}\right)$ is a diagonal matrix. Therefore, $\Phi$ and $\Psi$ are $2 \times 2$ matrices of the form

$$
\begin{array}{ll}
\Phi(\theta)=\left[\phi_{1}(\theta), \phi_{2}(\theta)\right], \quad \phi_{1}(\theta)=\mathrm{e}^{\mathrm{i} \omega_{k} \theta} v, \quad \phi_{2}(\theta)=\overline{\phi_{1}(\theta)}, \quad-1 \leqslant \theta \leqslant 0 \\
\Psi(s)=\left(\begin{array}{l}
\psi_{1}(s) \\
\psi_{2}(s)
\end{array}\right), \quad \psi_{1}(s)=\mathrm{e}^{-\mathrm{i} \omega_{k} s} u^{\mathrm{T}}, \quad \psi_{2}(s)=\overline{\psi_{1}(s)}, \quad 0 \leqslant s \leqslant 1,
\end{array}
$$

where the bar means complex conjugation, $u^{\mathrm{T}}$ is the transpose of $u$,

$$
u=\left(\begin{array}{l}
u_{1} \\
u_{2}
\end{array}\right)=\left(\begin{array}{c}
u_{1} \\
\frac{\mathrm{i} \omega_{k} \mathrm{e}^{\mathrm{i} \omega_{k}} u_{1}}{b_{1} \tau^{(k)}}
\end{array}\right) \in \mathbb{C}^{2}, \quad v=\left(\begin{array}{c}
v_{1} \\
v_{2}
\end{array}\right)=\left(\begin{array}{c}
1 \\
\frac{\mathrm{i} \omega_{k}}{a_{2} \tau^{(k)}}
\end{array}\right) \in \mathbb{C}^{2}
$$

with

$$
u_{1}=\frac{a_{2} b_{1} \tau^{(k)}}{\left(2 a_{2} b_{1} \tau^{(k)}-b_{2} \omega_{k}^{2}\right)+\left(a_{2} b_{1} \omega_{k} \tau^{(k)}+b_{2} \omega_{k}\right) \mathrm{i}} .
$$

Enlarge the phase space $C_{1}$ by considering the space $B C$ and using the decomposition $X_{t}=\Phi z(t)+y_{t}, z \in \mathbb{C}^{2}, y_{t} \in Q^{\prime}$. We decompose (3.8) as follows:

$$
\left.\begin{array}{l}
\dot{z}=B z+\Psi(0) F_{0}(\Phi z+y, \nu) \\
\dot{y}=A_{Q^{\prime}} y+(I-\pi) X_{0} F_{0}(\Phi z+y, \nu)
\end{array}\right\}
$$

Here and below we refer to $[\mathbf{9}]$ for results and explanations of some notation involved. Following the procedure of reducing normal form in $[\mathbf{9}]$, we consider the Taylor formula

$$
\begin{array}{r}
\Psi(0) F_{0}(\Phi z+y, \nu)=\frac{1}{2} g_{2}^{(1)}(z, y, \nu)+\frac{1}{3 !} g_{3}^{(1)}(z, y, \nu)+\text { h. o. t. } \\
(I-\pi) X_{0} F_{0}(\Phi z+y, \nu)=\frac{1}{2} g_{2}^{(2)}(z, y, \nu)+\frac{1}{3 !} g_{3}^{(2)}(z, y, \nu)+\text { h. o.t. }
\end{array}
$$

where $g_{j}^{(1)}(z, y, \nu), g_{j}^{(2)}(z, y, \nu)(j=2,3)$ are homogeneous polynomials in $(z, y, \nu)$ of degree $j$ with coefficients in $\mathbb{C}^{2}$ and $\operatorname{Ker} \pi$, respectively, and h. o. t. stands for higher-order 
terms. Thus in a finite-dimensional locally invariant manifold tangent to the invariant subspace $P$ of (3.9) at $x=0, \nu=0$, the normal form of (3.9) is given by

$$
\dot{z}=B z+\frac{1}{2} \bar{g}_{2}^{(1)}(z, 0, \nu)+\frac{1}{3 !} \bar{g}_{3}^{(1)}(z, 0, \nu)+\text { h. o.t. },
$$

where $\bar{g}_{2}^{(1)}, \bar{g}_{3}^{(1)}$ are the second- and third-order terms in $(z, \nu)$, respectively. Using the notations in $[\mathbf{9}]$, we have

$$
\bar{g}_{2}^{(1)}(z, 0, \nu)=\operatorname{Proj}_{\operatorname{Ker}\left(M_{2}^{\prime}\right)} g_{2}^{(1)}(z, 0, \nu)
$$

where

$$
\begin{gathered}
\operatorname{Ker}\left(M_{2}^{\prime}\right)=\operatorname{span}\left\{\left(\begin{array}{c}
z_{1} \nu \\
0
\end{array}\right),\left(\begin{array}{c}
0 \\
z_{2} \nu
\end{array}\right)\right\}, \\
g_{2}^{(1)}(z, 0, \nu)=\left(\begin{array}{c}
2 \mathrm{i} \frac{\omega_{k}}{\tau^{(k)}} u^{\mathrm{T}} v z_{1} \nu+a_{20} z_{1}^{2}+a_{11} z_{1} z_{2}+a_{02} z_{2}^{2} \\
-2 \mathrm{i} \frac{\omega_{k}}{\tau^{(k)}} \bar{u}^{\mathrm{T}} \bar{v} z_{2} \nu+\bar{a}_{02} z_{1}^{2}+\bar{a}_{11} z_{1} z_{2}+\bar{a}_{20} z_{2}^{2}
\end{array}\right) .
\end{gathered}
$$

We would like to point out that the coefficients $a_{20}, a_{11}$ and $a_{02}$ can be expressed in terms of the parameters in the original equations (3.8) by the algorithm of normal form. However, the explicit formulae for $a_{20}, a_{11}$ and $a_{02}$ are tediously long, since both $\Phi$ and $\Psi$ have quite complicated expressions, as shown above. Therefore, for the sake of convenience we prefer to use the notation $a_{20}, a_{11}$ and $a_{02}$ instead of their exact expressions in the following context.

To eliminate the non-resonant terms in the quadratic expressions $g_{2}^{(1)}(z, 0, \nu)$ we have to make a series of transformations of variables, which may change the coefficients of the cubic terms of $g_{3}^{(1)}(z, 0, \nu)$. From the canonical basis for $\operatorname{Ker}\left(M_{2}^{\prime}\right)$, we have

$$
\frac{1}{2} \bar{g}_{2}^{(1)}(z, 0, \nu)=\left(\begin{array}{c}
\mathrm{i} \frac{\omega_{k}}{\tau^{(k)}} u^{\mathrm{T}} v z_{1} \nu \\
-\mathrm{i} \frac{\omega_{k}}{\tau^{(k)}} \bar{u}^{\mathrm{T}} \bar{v} z_{2} \nu
\end{array}\right)
$$

Furthermore, recalling the operator $M_{3}^{\prime}$, we know that

$$
\operatorname{Ker}\left(M_{3}^{\prime}\right)=\operatorname{span}\left\{\left(\begin{array}{c}
z_{1}^{2} z_{2} \\
0
\end{array}\right),\left(\begin{array}{c}
z_{1} \nu^{2} \\
0
\end{array}\right),\left(\begin{array}{c}
0 \\
z_{1} z_{2}^{2}
\end{array}\right),\left(\begin{array}{c}
0 \\
z_{2} \nu^{2}
\end{array}\right)\right\} .
$$

However, the terms $O\left(|z| \nu^{2}\right)$ are irrelevant to determining the generic Hopf bifurcation. Hence we only need to compute the coefficient of $z_{1}^{2} z_{2}$. After some computations we find that the coefficient of $z_{1}^{2} z_{2}$ is

$$
c=\frac{\mathrm{i}}{2 \omega_{k}}\left(a_{20} a_{11}-2\left|a_{11}\right|^{2}-\frac{1}{3}\left|a_{02}\right|^{2}\right)+\frac{1}{2} a_{21},
$$


where $\frac{1}{2} a_{21}$ is the coefficient of the term $z_{1}^{2} z_{2}$ in $g_{3}^{(1)}(z, 0, \nu)$. Thus

$$
\frac{1}{3 !} \bar{g}_{3}^{(1)}(z, 0, \nu)=\left(\begin{array}{c}
c z_{1}^{2} z_{2} \\
\bar{c} z_{1} z_{2}^{2}
\end{array}\right)+O\left(|z| \nu^{2}\right) \text {. }
$$

The normal form (3.10) relative to $P$ can be written in real coordinates $(x, y)$, through the change of variables $z_{1}=x-\mathrm{i} y, z_{2}=x+\mathrm{i} y$. Then, using the polar coordinates $(r, \theta)$, $x=r \cos \theta, y=r \sin \theta$, this normal form becomes

$$
\begin{aligned}
& \dot{r}=c_{1} \nu r+c_{2} r^{3}+O\left(\nu^{2} r+|(r, \nu)|^{4}\right), \\
& \dot{\theta}=-\omega_{k}+O(|(r, \nu)|),
\end{aligned}
$$

where

$$
c_{1}=\operatorname{Re}\left(\mathrm{i} \frac{\omega_{k}}{\tau^{(k)}} u^{\mathrm{T}} v\right), \quad c_{2}=\operatorname{Re}(c) .
$$

Therefore, these arguments lead to the following theorem.

Theorem 3.3. If $c_{2} \neq 0$ and $\tau^{(k)}>0$, then (3.8) exhibits a generic Hopf bifurcation. The periodic orbits of (3.8) bifurcating from the origin and $\nu=0$ satisfy

$$
r(t, \nu)=\sqrt{-\frac{c_{1} \nu}{c_{2}}}+O(\nu), \quad \theta(t, \nu)=-2 \omega_{k} t+O\left(|\nu|^{1 / 2}\right)
$$

so that

(i) if $c_{1} c_{2}<0$ (respectively, $c_{1} c_{2}>0$ ), there exists a unique non-trivial periodic orbit in a neighbourhood of $r=0$ for $\nu>0$ (respectively, $\nu<0$ ) and no non-trivial periodic orbits for $\nu<0(\nu>0$, respectively); and

(ii) the non-trivial periodic solutions in the centre manifold are stable if $c_{2}<0$ and unstable if $c_{2}>0$.

As an example of the preceding results, we now consider

$$
\left.\begin{array}{l}
\dot{x}(t)=\tau\left(x(t)(1-x(t))-\frac{2 x(t) y(t)}{y(t)+x(t)}\right), \\
\dot{y}(t)=\tau y(t)\left(-\sqrt{2}+\frac{2 x(t-1)}{y(t-1)+x(t-1)}\right)
\end{array}\right\}
$$

in a fixed phase space $C_{1}=C\left([-1,0] ; R_{+}^{2}\right)$.

The system (3.11) has a unique interior equilibrium $E^{*}=\left(\sqrt{2}-1,(\sqrt{2}-1)^{2}\right)$. The purpose here is to consider the dynamics of (3.11) in a neighbourhood of $E^{*}$ at a bifurcation point of the parameter $\tau$. In the following, we shall apply Theorems 3.2 and 3.3 to (3.11) and obtain the following theorem.

Theorem 3.4. Let $\omega_{1} \in(2 \pi, 3 \pi)$ and $\tau_{1}$ be defined by

$$
\omega_{1}=\arccos \frac{\sqrt{5}-1}{2}+2 \pi, \quad \tau_{1}=\omega_{1} \frac{\sqrt{\sqrt{5}-1}}{2-\sqrt{2}} .
$$


Then for $\tau=\tau_{1}, \pm \mathrm{i} \omega_{1}$ are simple roots of the characteristic equation of (3.11) at $E^{*}$ and the remaining roots have non-zero real parts. Furthermore, for (3.11) there is a subcritical Hopf bifurcation at $\tau=\tau_{1}$, with the non-trivial periodic orbit being unstable in the centre manifold, and the centre manifold is unstable.

Proof. According to Theorem 3.2, we get immediately, when

$$
\tau=\tau_{k}=\omega_{k} \frac{\sqrt{\sqrt{5}-1}}{2-\sqrt{2}}
$$

that $\pm \mathrm{i} \omega_{k}$ are simple roots of the characteristic equation of (3.11) at $E^{*}$ and the remaining roots have non-zero real parts, where $\omega_{k}=\arccos \frac{1}{2}(\sqrt{5}-1)+2 k \pi, k=0,1,2, \ldots$ More precisely, when $\tau \in\left(0, \tau_{0}\right)$, all roots of the characteristic equation of (3.11) at $E^{*}$ have negative real parts, (3.11) then has a locally asymptotically stable equilibrium $E^{*}$; when $\tau=\tau_{0}, \pm \mathrm{i} \omega_{0}$ are simple roots of the characteristic equation of (3.11) at $E^{*}$ and the remaining roots have negative real parts, (3.11) then has a stable centre manifold; when $\tau=\tau_{k}, \pm \mathrm{i} \omega_{k}$ are simple roots of the characteristic equation of (3.11) and the remaining roots have non-zero real parts, (3.11) then has an unstable centre manifold for $k=$ $1,2, \ldots$

We are interested in studying the behaviour of periodic solutions bifurcating from $\tau=\tau_{1}$. Taking $\nu=\tau-\tau_{1}$, the system (3.11), in the notation introduced prior to Theorem 3.3, becomes

$$
\dot{X}(t)=\tau_{1} L\left(X_{t}\right)+F_{0}\left(X_{t}, \nu\right),
$$

where $F_{0}(\varphi, \nu)=\nu L(\varphi)+\left(\tau_{1}+\nu\right) F(\varphi)$ for $\varphi=\left(\varphi_{1}, \varphi_{2}\right) \in C_{1}$,

$$
\begin{aligned}
& L(\varphi)=\left(\begin{array}{c}
-\varphi_{2}(0) \\
(\sqrt{2}-1)^{2} \varphi_{1}(-1)+(-\sqrt{2}+1) \varphi_{2}(-1)
\end{array}\right) \\
& F(\varphi)=\left(\begin{array}{c}
F_{1}\left(\varphi_{1}(0), \varphi_{2}(0)\right) \\
F_{2}\left(\varphi_{2}(0), \varphi_{1}(-1), \varphi_{2}(-1)\right)
\end{array}\right) .
\end{aligned}
$$

We can compute the vectors $u$ and $v$ according to their expressions given before Theorem 3.3. Omitting these complicated expressions, we obtain the numerical results directly by means of the software MATHEMatica:

$$
u=\left(\begin{array}{l}
u_{1} \\
u_{2}
\end{array}\right)=\left(\begin{array}{c}
-0.05827-0.06901 \mathrm{i} \\
0.27164+0.05601 \mathrm{i}
\end{array}\right), \quad v=\left(\begin{array}{l}
v_{1} \\
v_{2}
\end{array}\right)=\left(\begin{array}{c}
1 \\
-0.52689 \mathrm{i}
\end{array}\right) .
$$

Following the procedure of reducing normal form, we can get the coefficients in the case when $\nu=0$ by means of the software MathematicA:

$$
\begin{array}{ll}
a_{20}=0.765562-0.380496 \mathrm{i}, & a_{11}=0.345173-0.291445 \mathrm{i}, \\
a_{02}=0.184747-1.41851 \mathrm{i}, & a_{21}=0.28828+0.85934 \mathrm{i} .
\end{array}
$$


Recalling some expressions introduced before Theorem 3.3,

$$
c=\frac{\mathrm{i}}{2 \omega_{k}}\left(a_{20} a_{11}-2\left|a_{11}\right|^{2}-\frac{1}{3}\left|a_{02}\right|^{2}\right)+\frac{1}{2} a_{21}, \quad c_{1}=\operatorname{Re}\left(\mathrm{i} \frac{\omega_{k}}{\tau^{(k)}} u^{\mathrm{T}} v\right), \quad c_{2}=\operatorname{Re}(c),
$$

we have

$$
c_{1}=0.111771, \quad c_{2}=0.168797 \text {. }
$$

Therefore, Theorem 3.3 implies the occurrence of a subcritical Hopf bifurcation at the point $\tau=\tau_{1}$ from equilibrium $E^{*}$ with the associated periodic solution being unstable in the centre manifold.

\section{Discussion}

It is interesting to compare our local stability results with the global results in [4]. We make the local stable analysis of the interior equilibrium depending on all parameters and bifurcation analysis by choosing time delay $\tau$ as a bifurcation parameter. Our analysis supports their results (i.e. the global stability of the interior equilibrium must require conditions on $\tau$ ) and also complements their results in the sense that we give all conditions depending on all parameters in (1.2) such that the interior equilibrium is not globally stable, and show the sufficient conditions which guarantee the existence and stability of the non-trivial periodic solution of (1.2). Our analysis indicates that the dynamics of the ratio-dependent predator-prey system with time delay can be much more complicated than we may have expected. It is still interesting and challenging to systematically describe the global dynamics of the model for all parameters by means of the local properties of the interior equilibrium.

Acknowledgements. The research is supported by the National Natural Science Foundations of China (no. 10071027) and the Laboratory of Nonlinear Analysis. The authors are grateful to the anonymous referee for helpful comments and valuable suggestions.

\section{References}

1. H. R. Akcakaya, Population cycles of mammals: evidence for a ratio-dependent predation hypothesis, Ecol. Monogr. 62 (1992), 119-142.

2. R. ARditi And L. R. GinzBuRG, Coupling in predator-prey dynamics: ratio-dependence, J. Theor. Biol. 139 (1989), 311-326.

3. R. Arditi, L. R. Ginzburg and H. R. AkCakaya, Variation in plankton densities among lakes: a case of ratio-dependent models, Am. Naturalist 138 (1991), 1287-1296.

4. E. BERETTA AND Y. KUANG, Global analyses in some delayed ratio-dependent predatorprey systems, Nonlin. Analysis 32 (1998), 381-408.

5. A. A. BerRyman, The origins and evolution of predator-prey theory, Ecology 75 (1992), 1530-1535.

6. F. Brauer, Absolute stability in delay equations, J. Diff. Eqns 69 (1987), 185-191.

7. K. L. CoOke And Z. Grossman, Discrete delay, distributed delay and stability switches, J. Math. Analysis Applic. 86 (1982), 592-627.

8. J. Dieudonné, Foundations of modern analysis (Academic, 1960). 
9. T. FARIA AND L. T.MAGAlhãEs, Normal forms for retarded functional differential equations with parameters and applications to Hopf bifurcation, J. Diff. Eqns 122 (1995), 181-200.

10. A. P. Gutierrez, The physiological basis of ratio-dependent predator-prey theory: a metabolic pool model of Nicholson's blowflies as an example, Ecology 73 (1992), 15521563.

11. C. Jost, O. Arino And R. Arditi, About deterministic extinction in ratio-dependent predator-prey models, Bull. Math. Biol. 61 (1999), 19-32.

12. Y. KUANG, Delay differential equations with applications in population dynamics (Academic, 1993).

13. Y. KUANG, Rich dynamics of Gause-type ratio-dependent predator-prey system, Fields Inst. Commun. 21 (1999), 325-337.

14. Y. KUANG AND E. BERETTA, Global qualitative analysis of a ratio-dependent predatorprey system, J. Math. Biol. 36 (1998), 389-406.

15. P. LUNDBERG AND J. M. FRYXELL, Expected population density versus productivity in ratio-dependent and prey-dependent models, Am. Naturalist 146 (1995), 153-161.

16. M. L. Rosenzweig, Paradox of enrichment: destabilization of exploitation ecosystems in ecological time, Science 171 (1971), 385-387.

17. D. Xiao And S. RuAn, Global dynamics of a ratio-dependent predator-prey system, $J$. Math. Biol. 43 (2001), 268-290. 